\title{
Axonal TDP-43 aggregates in sporadic amyotrophic lateral sclerosis
}

\author{
Tomoya Onozato $^{1,2}$, Asa Nakahara ${ }^{1}$, Emi Suzuki-Kouyama ${ }^{1}$, Akiyo Hineno ${ }^{3}$, \\ Takuji Yasude ${ }^{3}$, Toshitsugu Nakamura ${ }^{4}$, Hiroyuki Yahikozawa ${ }^{5}$, Masahide \\ Watanabe $^{6}$, Katsuhiko Kayanuma ${ }^{7}$, Hideo Makishita ${ }^{8}$, Shinji Ohara ${ }^{9}$, Takao \\ Hashimoto $^{10}$, Kayoko Higuchi ${ }^{11}$, Toshiaki Sakai ${ }^{12}$, Kouji Asano ${ }^{13}$, Tomoyo \\ Hashimoto $^{1,14}$, Hiroyuki Kanno ${ }^{15}$, Jun Nakayama ${ }^{16}$, Kiyomitsu Oyanagi ${ }^{1}$
}

${ }^{1}$ Division of Neuropathology, Department of Brain Disease Research, Shinshu University School of Medicine, Nagano, Japan

${ }^{2}$ Safety Research Laboratory of Research and Development, Kissei Pharmaceutical Co., Ltd., Nagano, Japan

${ }^{3}$ Department of Neurology, Suwa Red Cross Hospital, Nagano, Japan

${ }^{4}$ Department of Pathology, Suwa Red Cross Hospital, Nagano, Japan

${ }^{5}$ Department of Neurology, Nagano Red Cross Hospital, Nagano, Japan

${ }^{6}$ Department of Pathology, Nagano Red Cross Hospital, Nagano, Japan

${ }^{7}$ Department of Neurology, Ina Central Hospital, Nagano, Japan

${ }^{8}$ Department of Neurology, Hokushin General Hospital Nagano, Japan

${ }^{9}$ Department of Neurology, Matsumoto Medical Center, Nagano, Japan

${ }^{10}$ Department of Neurology, Aizawa Hospital, Nagano, Japan

${ }^{11}$ Section of Anatomic Pathology, Aizawa Hospital, Nagano, Japan

${ }^{12}$ Department of Neurology, Nagano Matsushiro General Hospital, Nagano, Japan

${ }^{13}$ Department of Pathology, Suwa Chuo Hospital, Nagano, Japan 
${ }^{14}$ Department of Neurology, University of Occupational and Environmental Health, Fukuoka, Japan

${ }^{15}$ Department of Pathology, Shinshu University School of Medicine, Nagano, Japan

${ }^{16}$ Department of Molecular Pathology, Shinshu University Graduate School of Medicine, Nagano, Japan

Correspondence to: Kiyomitsu Oyanagi, $\mathrm{MD}, \mathrm{PhD}$

Division of Neuropathology,

Department of Brain Disease Research,

Shinshu University School of Medicine,

Asahi 3-1-1, Matsumoto, Nagano 390-8621, Japan

Phone: $+81-263-37-3185 \quad$ Fax: $+81+263-37-3186$

e-mail: k123ysm@shinshu-u.ac.jp

\section{Keywords:}

ALS; axonal aggregate; TDP-43; topography; synapse

\section{Running title}

Axonal TDP-43 aggregates in sporadic ALS

Word count: 5,491, Figures 4, Tables 3

\section{Abstract}

Aims: Axonal aggregates of phosphorylated (p-) transactive response DNA-binding protein $43 \mathrm{kDa}$ (TDP-43) in sporadic amyotrophic lateral sclerosis (sALS) were examined in relation to propagation of the protein in the nervous system. Methods: Brains, spinal cords and muscles of Japanese patients with 
sALS and control subjects were examined immunohistochemically using formalin-fixed

paraffin-embedded specimens with special reference to the topographic distribution, microscopic features, presynaptic aggregates and correlation between the aggregates in axons and the clinical course. Results: 1) Aggregates of p-TDP-43 were frequently present in axons of the hypoglossal and facial nerve fibers and the spinal anterior horn cells. 2) Aggregates of p-TDP-43 in the axons showed two characteristic microscopic features - dash-like granuloreticular aggregates (GRAs) and massive aggregates (MAs). 3) MAs were surrounded by p-neurofilaments, but p-neurofilament immunnoreactivity decreased at the inside of axons with GRAs. 4) Patients showing MAs and GRAs had a relatively shorter clinical course than patients without the aggregates. 5) Some neurons in the red nucleus in patients were surrounded by synapses containing p- and p-independent (i)-TDP-43, and almost all neurons had lost their nuclear TDP-43 immunoreactivity; $17 \%$ of those neurons in the red nucleus also had TDP-43-immunopositive neuronal cytoplasmic inclusions, but no postsynaptic p-TDP-43 deposition was evident. Conclusions: There are two types of axonal p-TDP-43 aggregates, MAs and GRAs, located predominantly in the facial and hypoglossal nuclei and anterior horn cells. These aggregates may influence the function of neurons, and presynaptic aggregates of the protein induce loss of p-i-TDP-43 in the nuclei of postsynaptic neurons.

\section{Abbreviations:}

$\mathrm{Ab}=$ antibody $\mathrm{AHC}=$ anterior horn cell; $\mathrm{ALS}=$ amyotrophic lateral sclerosis; ALS-D = ALS with dementia; bicep = biceps; delt = deltoid; diaph=diaphragm; FTLD =frontotemporal lobar degeneration; GRA = granuloreticular aggregate; hypogl = hypoglossal; iliops = iliopseas; intramuscul = intramuscular; int cost $=$ intercostal; ir = immunoreactive; $\mathrm{MA}=$ massive aggregate; $\mathrm{MBP}=$ myelin basic protein; med $\mathrm{obl}=$ medulla oblongata; - moderate; $\mathrm{NA}=$ not available; $\mathrm{NCI}=$ neuronal cytoplasmic inclusion; ne $=$ not examined $; \mathrm{NF}=$ neurofilament $\mathrm{n}-\mathrm{p}=$ nonphosphorylated $\mathrm{p}=$ phosphorylated $\mathrm{p}-\mathrm{i}=$ phosphorylation independent; $\mathrm{RI}=$ round inclusion; $\mathrm{PN}=$ perineuronal; $\mathrm{PNSA}=$ perineuronal synaptic 
aggregate precentr $=$ precentral; pyramid $=$ pyramidal $;$ ret form $=$ reticular formation; sALS $=$ sporadic amyotrophic lateral sclerosis; sev $=$ severe; $\mathrm{SG}=$ small granule; $\mathrm{SI}=$ skein-like inclusion; $\mathrm{SYP}=$ synaptophysin; TDP-43 = transactive response DNA-binding protein $43 \mathrm{kDa}$; WM = convolutional white matter; $-=$ absent

\section{Introduction}

Sporadic amyotrophic lateral sclerosis (sALS) is an adult onset neurodegenerative disease of humans characterized by degeneration of mainly the upper and lower motor neurons. Bunina bodies and deposition of phosphorylated (p-) transactive response DNA-binding protein $43 \mathrm{kDa}$ (p-TDP-43) in the cytoplasm of the neurons, in addition to degeneration of the corticospinal tracts and loss of spinal anterior horn cells, are the major pathological hallmarks of the disease [1 - 5].

Nonphosphorylated (n-p-) TDP-43 is normally present in the nucleus, although the protein is synthesized in the cytoplasmic compartment and is capable of shuttling between the nucleus and the cytoplasm. Although the physiological function of n-p-TDP-43 is still incompletely characterized, it has been reported that the protein has several roles in the regulation of gene expression and RNA function [6 $-8]$. TDP-43 in the brains of patients with sALS and frontotemporal lobar degeneration (FTLD) is often hyperphosphorylated, cleaved, ubiquitinated, mislocalized and poorly soluble in the cytoplasm $[6,8-10]$. Recent papers have concluded that p-TDP-43 is probably not toxic [7, 9]. Diseases characterized by p-TDP-43 deposition are referred to as TDP-43 proteinopathies [1, 11], and the topographic distribution of p-TDP-43 in sALS is classified as "ALS" or “ALS-D" in TDP-43 spectrum [12], type "1" or "2" [13] or as stage "1" to "4" [14].

Regarding the spreading of the pathognomonic proteins in sALS, FTLD with TDP pathology, Parkinson's disease, and Alzheimer's disease, it has been suggested that p-TDP-43, p-alpha synuclein, 
beta amyloid and p-tau may exhibit prion-like propagation in the brain [14 - 17]. However, there have been no reports of cell-to-cell transmission of these proteins in the nervous system of patients. If p-TDP-43 spreads in the same manner as a prion in the nervous system of patients with sALS, it is conceivable that it would exist in axons or dendrites, and pass through the synapse or plasma membrane. With regard to p-TDP-43 in the axons of patients with sALS, only a few papers have reported dash-like accumulation in axons of the hypoglossal nerve and anterior horn cells [14, 18], although the pathological significance of this phenomenon remains unclear.

In the present study, we examined the brains, spinal cords, tongues and skeletal muscles of 19 Japanese patients with sALS and three control subjects immunohistochemically using formalin-fixed paraffin-embedded specimens of nervous tissue and muscle with special reference to p-TDP-43 in the neuronal cytoplasm, axons and synapses, and considered the pathological significance of the protein in axons and its correlation with the clinical course or symptoms of sALS.

\section{Materials and Methods}

\section{Subjects and clinical characterization}

We examined autopsy specimens of the brain, spinal cord, tongue and skeletal muscles, such as the biceps brachii, deltoid, iliopsoas or intercostal muscle, from 19 Japanese patients who had been clinically diagnosed and neuropathologically verified as having sALS (11 males and eight females; age at autopsy, 59-88 years; mean \pm standard deviation $70.2 \pm 9.1$ years; disease onset, $68.1 \pm 9.1$ years; clinical course, $24.2 \pm 16.9$ months). In order to observe axonal aggregates, we selected patients in whom a relatively large number of lower motor neurons remained and demonstrated relatively many p-TDP-43 immunoreactive (-ir) aggregates after clinical courses of between 6 and 72 months. Three age-matched autopsied Japanese subjects without neurological symptoms or any pathologically remarkable intracranial lesions (three males; age at autopsy 65-74 years; mean \pm standard deviation $69.3 \pm 4.5$ years) were used 
as controls. Neither the patients nor the controls had significantly low blood pressure, and none had a history of cardiac arrest or anti-cancer therapy. Table 1 summarizes the clinical and pathological findings of the patients.

All subjects provided written informed consent by their families to participate in the study, and the study was approved by the Institutional Review Board of Shinshu University School of Medicine (Approval No. 1565).

\section{Preparation of brain tissue and muscle for light microscopic examination and immunohistochemistory}

Formalin-fixed paraffin-embedded 6 - $\mu$ m-thick serial coronal sections of the frontal, parietal, temporal and occipital lobes, transverse sections of the brainstem and spinal cord, and transverse or sagittal sections of the cerebellum were subjected to neuropathological examination after hematoxylin and eosin (HE), Klüver-Barrera and periodic acid-Schiff staining. Immunohistochemistry was performed with primary antibodies (Abs) against p-TDP-43, phosphorylation independent (p-i-) TDP-43, ubiquitin, p62, myelin basic protein (MBP), synaptophysin (SYP), p-alpha synuclein, p-neurofilament (p-NF) and p-tau (Table 2).

In addition, formalin-fixed paraffin-embedded 6 - $\mu$ m-thick serial sections of the tongue and skeletal muscles, such as the biceps brachii, deltoid, iliopsoas and intercostals muscles, from eight sALS patients (Nos. 1, 2, 6, 9, 13, 14, 15 and 17) were subjected to pathological examination.

Immunohistochemical staining was performed using the avidin-biotin-peroxidase complex method (Vectastain ABC Elite kit; Vector, Burlingame, CA, USA). Non-specific binding of the avidin-biotin-peroxidase complex $(\mathrm{ABC})$ system reagents was blocked by pretreating the sections with $0.3 \%$ hydrogen peroxide in methanol and a normal blocking serum. The sections were then incubated with the required primary $\mathrm{Ab}$ overnight at $4^{\circ} \mathrm{C}$. They were then rinsed in phosphate-buffered saline 
containing Triton X-100 (pH 7.6) (PBST) and incubated for 1 hour with the secondary reagent containing a biotinylated anti-rabbit or anti-mouse $\operatorname{IgG} \mathrm{Ab}$ (diluted $1: 200$ ) at $37^{\circ} \mathrm{C}$, and finally with the $\mathrm{ABC}$ solution for 1 hour at room temperature. The sections were subjected to a peroxidase reaction with $30 \mu \mathrm{L}$ ImmPACT ${ }^{\mathrm{TM}}$ DAB Chromogen Concentrate (Vector) (diluted 1:2 with $50 \mathrm{mmol} / \mathrm{L}$ Tris- $\mathrm{HCl}(\mathrm{pH}$ 7.6)) in 1 $\mathrm{mL}$ ImmPACT ${ }^{\mathrm{TM}}$ Diluent (Vector) for 2 minutes at room temperature. After stopping the reactions, the sections were rinsed in tap water, and then counterstained with hematoxylin.

Antigenicity for p-TDP-43 (rabbit polyclonal) and p-NF immunohistochemistry was increased by boiling the sections in $0.01 \mathrm{~mol} / \mathrm{L}$ citrate buffer solution ( $\mathrm{pH} 7.6)$ in a microwave oven $(750 \mathrm{~W}, 25$ minutes); for p-TDP-43 (mouse monoclonal), p-i-TDP-43, ubiquitin, p62, MBP and SYP, the sections were autoclaved $\left(121^{\circ} \mathrm{C}, 20\right.$ minutes) in $0.01 \mathrm{~mol} / \mathrm{L}$ citrate buffer solution $(\mathrm{pH} 6.0)$, and for p-alpha synuclein, the sections were incubated with $99 \%$ formic acid for 5 minutes at room temperature.

We performed double immunohistochemistry for p-TDP-43 with MBP and p-TDP-43 with p-NF in the medulla oblongata and lumbar spinal cord. The sections were first boiled in a microwave oven, then anti-p-TDP-43 Ab (1:10000) was initially applied and the sections were subjected to peroxidase reaction with $30 \mu \mathrm{L}$ ImmPACT ${ }^{\mathrm{TM}} \mathrm{DAB}$ Chromogen concentrate (Vector, Burlingame, CA, USA) (diluted 1:2 in 50 $\mathrm{mM}$ Tris- $\mathrm{HCl}(\mathrm{pH} 7.6))$ in $1 \mathrm{~mL}$ ImmPACT TM Diluent (Vector) for 2 minutes at room temperature, in accordance with the single staining procedure. After pretreatment in Denaturing Solution (A:B = 1:2) (Biocare Medical, Concord, CA, USA) for 3 minutes, the sections were incubated in PBST. Anti-MBP Ab $(1: 400)$ or $\mathrm{p}-\mathrm{NF} \mathrm{Ab}(1: 1000)$ was added and the sections were then incubated for 17 hours at $4^{\circ} \mathrm{C}$. The sections were washed three times in PBST, and then incubated with secondary biotinylated Ab for 1 hour at $37^{\circ} \mathrm{C}$. They were then washed three times in PBST, followed by application of the $\mathrm{ABC}$ method and reaction with Vina Green Chromogen (Biocare Medical).

As Ab controls, the primary antisera were either omitted or replaced with normal rabbit or mouse serum. Several specimens of neural or non-neuronal tissue from the patients served as positive or negative 
tissue controls. The preparations were examined by light microscopy.

\section{Confocal immunofluorescence examination}

We performed confocal immunofluorescence observation for p-TDP-43 with p-NF and p-TDP-43 with ubiquitin in the medulla oblongata from sALS patients Nos. 1 and 6 and the lumbar spinal cord from sALS patients Nos. 1, 2 and 6, and for anti-p-TDP-43 polyclonal Ab with anti-SYP Ab in the midbrain from ALS patient No. 1. Formalin-fixed paraffin-embedded 6- $\mu$ m-thick serial sections were autoclaved, and then incubated in normal goat serum for 30 minutes. The sections were then incubated with the anti-p-TDP-43 polyclonal Ab (1:50), anti-p-NF antibody (1:200), anti-p-TDP-43 monoclonal Ab (1:200), anti-ubiquitin Ab (1:100), anti-p-TDP-43 polyclonal Ab (1:200) and anti-SYP Ab (1:50) for 2 hours. The sections were then rinsed in PBS and incubated for 1 hour with the secondary reagent containing Abs: Alexa Fluor 546 goat anti-rabbit IgG (diluted 1:200; Molecular Probes, Eugene, Oregon, USA) and Alexa Fluor 488 goat anti-mouse IgG (diluted 1:200; Molecular Probes). The sections were then immersed in autofluorescence eliminator reagent (Millipore, Temecula, CA, USA) for 1 minute at room temperature to remove any of the autofluorescence pigment lipofuscin that had accumulated. These sections were observed using a confocal laser microscope (LSM5; Carl Zeiss, Oberkochen, Germany).

\section{Immunoelectron microscopic examination of synaptic aggregates}

We performed immunoelectron microscopy using formalin-fixed paraffin-embedded $10-\mu m$-thick serial sections of the midbrain from ALS patient No. 1. These sections were immunostained with anti-p-TDP-43 monoclonal $\mathrm{Ab}$ in the same manner, then fixed in 4\% paraformaldehyde and $1 \%$ glutaraldehyde in $0.1 \mathrm{M}$ phosphate buffer ( $\mathrm{pH} 7.4$ ) overnight, followed by immersion in $0.1 \mathrm{M}$ phosphate buffer for 15 minutes three times. The sections were post-fixed in $1 \%$ osmium tetroxide in $0.1 \mathrm{M}$ phosphate buffer for 1 hour, and then embedded in Epok 812 (Oken Shoji Co., Tokyo, Japan). Ultrathin 
sections $90 \mathrm{~nm}$ thick were double-stained with uranyl acetate for 15 minutes and lead citrate for 5 minutes, and then examined with an electron microscope (JEM-1400; JEOL, Tokyo, Japan).

\section{Semiquantitative examination of skeletal musele atrophy}

Severity of skeletal muscle atrophy was classified into four grades based on light microscopic observation on HE stained formalin-fixed paraffin-embedded sections. Those are; no = normal; mild = number of angulated atrophic muscle fibers being less than half of that of muscle fibers examined; moderate = number of angulated atrophic muscle fibers being more than half of that of muscle fibers examined and severe $=$ most of muscle fibers being atrophic with marked fatty infiltration.

\section{Results}

\section{Morphologic and immunohistochemical features of axonal p-TDP-43 aggregates}

There were no p-TDP-43-ir aggregates in control subjects, but all sALS patients showed p-TDP-43-ir neuronal cytoplasmic inclusions (NCIs) such as skein-like inclusions (SIs), small granules (SGs) or round inclusions (RIs) in the motor cortex, facial and hypoglossal nuclei, anterior horn or red nucleus [5] (Table 3). Two patterns of axonal p-TDP-43-ir aggregates were observed (Figure 1A-L): dash-like granuloreticular aggregates (GRAs) (Figure 1A-G) and massive aggregates (MAs) (Figure 1H-L). GRAs appeared as fine granular or reticular patterns about $1 \mu \mathrm{m}$ in diameter within the circumference of the axons, and intermingled with p-NF (Figure 1A-G). The finding was similar to that reported by Braak et al. [18]. p-NF immunoreactivity was lost in many portions at the inside the axons with GRAs (Figure 1F, G). MAs were ovoid or gourd-shaped measuring less than $10 \mu \mathrm{m}$ in longest diameter and less than $5 \mu \mathrm{m}$ in shortest diameter (Figure 1H-L). MAs were circumscribed by MBP-ir myelin and surrounded by p-NF (Figure 1I-L).

Both GRAs and MAs were also immunoreactive for p-i-TDP-43, ubiquitin and p62 (Figure 1M-Q), 
but negative for $\mathrm{p}$-alpha synuclein, $\mathrm{p}-\mathrm{NF}, \mathrm{MBP}$ and $\mathrm{p}$-tau.

Though areas of many axonal p-TDP-43 aggregates have relatively many glial p-TDP-43 inclusions, close localization or direct continuity between axonal and glial p-TDP-43 inclusions were not observed.

\section{Topographic localization of p-TDP-43-ir structures}

p-TDP-43-ir neuronal cytoplasmic inclusions (NCIs) were observed in the motor cortex, facial and hypoglossal nuclei, anterior horn or red nucleus in all patients (Table 3).

p-TDP-43-ir GRAs in axons were observed at the precentral gyri in two out of 13 patients, at the lateral corticospinal tracts in the lumbar segments in one out of 19 patients, at the intramedullary facial nerve in four out of eight patients, at the intramedullary hypoglossal nerve in six out of 15 patients, and at the intramedullary axons of anterior horn cells in nine out of 19 patients. In addition, GRAs were observed in the axons of the anterior root in five (Nos. 1, 2, 6, 12 and 13) out of 18 patients and the intramuscular nerves in the tongue of one (No. 1) out of five patients. MAs were observed in the axons of the intramedullary facial nerve in one out of eight patients examined, at the intramedullary hypoglossal nerve in two out of 15 patients, and the intramedullary axons of anterior horn cells in three out of 19 patients (Table 3). In addition, GRAs and MAs were found in axons in the dorsal vagal nucleus in patient No.1. There were no p-TDP-43-ir axonal aggregates in the spinal dorsal funiculus or dorsal root.

p-TDP-43-ir glial inclusions are not shown in Table 3, although they were widely observed in the patients with sALS.

MAs were located within several hundred micrometers from the neurons or origin, whereas GRAs were located within a few millimeters (Figure 2). All patients showing MAs in axons also had GRAs in axons and NCIs, and had relatively short clinical courses (Figure 3).

\section{Axonal p-TDP-43-ir aggregates, TDP-43 Spectrum [12], Patterns [13] and Stages}




\section{[14] in relation to muscle atrophy and clinical course}

GRAs were observed in 10 of the 19 patients, of whom four were ALS of TDP-43 Spectrum [12] and Pattern 1 [13] and six showed ALS-D in TDP-43 Spectrum and Pattern 2; three patients were at Stage 1 or $1 / 2$ [14], four were at Stage 2, and three were at Stage 4. MAs were observed in three of the 19 patients, of whom three showed ALS-D in TDP-43 Spectrum and Pattern 2; one patient was at Stage 2 and two were at Stage 4.

The duration of illness in the patients with GRAs ranged from 6 to 31 months (mean \pm standard deviation; $16.3 \pm 9.2$ months), and that in the patients with MAs ranged from 6 to 11 months (mean \pm standard deviation; $7.7 \pm 2.9$ months). It was difficult to precisely examine the relation between the presence or absence of GRAs or MAs in the lower motor neuron axons and the severity of atrophy of the skeletal muscles, for the reason that the number of patients with skeletal muscles examined was small, and that some patients showed severe muscle atrophy and some showed mild one though they had GRAsor MAs in the lower motor neuron axons (Table 3).

\section{Morphological and quantitative features of perineuronal synaptic aggregates of p-TDP-43}

p-TDP-43-ir perineuronal synaptic aggregates (PNSA) measuring 2-5 $\mu \mathrm{m}$ in diameter were observed around neurons in the red nuclei in three out of 12 patients examined (Figure 4A), and in the reticular formation of the medulla oblongata in one out of 15 patients examined (Table 3). PNSA were immunopositive for p-i-TDP-43 (Figure 4B), ubiquitin and p62 (data not shown). Immunofluorescence confocal microscopy showed that p-TDP-43-ir PNSA were surrounded by SYP-ir structures (Figure 4C-F). Immunoelectron microscopy demonstrated p-TDP-43-ir PNSA in pre-synapses, appearing as 50-80-nm granular bodies (Figure 4G, H). There were no p-TDP or p-i-TDP-43-immunopositive aggregates in the post-synapses (Figure 4C-F). Medial predominance of neurons with p-TDP-ir PNSA 
was evident in the red nucleus (Figure 4I)

Immunohistochemistry for p-i-TDP-43 in the red nucleus of patient No. 1 demonstrated that 13 out of 78 neurons (17\%) with TDP-43-ir PNSA had intracytoplasmic TDP-43 deposition, and that 77 neurons (99\%) had lost nuclear TDP-43. Three out of 98 neurons (3\%) without TDP-43-ir PNSA in the red nucleus had intracytoplasmic TDP-43 deposition, and 88 neurons (90\%) retained nuclear TDP-43 (Figure 4B). Immunohistochemistry for p-TDP-43 demonstrated that four out of six neurons $(67 \%)$ with p-TDP-43-ir PNSA in the reticular formation of patient No. 1 had intracytoplasmic p-TDP-43 deposition.

\section{Discussion}

Dash-like deposits of p-TDP-43 have been reported in the axons of the hypoglossal nucleus and anterior horn cells in patients with sALS $[14,18,19]$. The finding is similar to GRAs reported in the present study. The present study revealed for the first time massive aggregates (MAs) of the protein in the axons of the facial and hypoglossal nuclei and anterior horn cells, in addition to GRAs. Immunopositivity of the MAs and GRAs for ubiquitin and p62 indicated similarities to the NCIs in the lower motor neurons in sALS [1, 20]. GRAs exist within the circumference of the axons, and MAs are located in the central part of the axons. GRAs close to the axon plasma membrane might interfere with depolarization, and thus disturb conduction, whereas MAs in the central part of the axon might disturb axonal flow. In the present study, it is unclear whether or not these pathologic aggregates were correlated with the severity of atrophy in themuscles examined. But it is probable that these aggregates had influenced muscle contraction and fasciculation.

MAs were located within several hundred micrometers from the neurons or origin, whereas GRAs were located within a few millimeters. Those differences in distance from the neuron cytoplasm may indicate a difference in the mechanisms of MA and GRA formation.

GRAs were present in patients with clinical courses of less than 31 months, and MAs in patients 
with clinical courses of less than 11 months; patients with clinical courses exceeding 11 months or 31 months did not have MAs. These findings raise two possibilities: 1) there are some sALS patients with or without axonal aggregates, and patients with MAs or GRAs die within six to 31 months, or 2) MAs and GRAs appear within a period of several months to 31 months, then disappear.

GRAs were observed in patients with ALS and ALS-D in TDP-43 Spectrum [12], patterns 1 and 2 [13], and at stages 1 to 4 [14] but MAs were present in patients with TDP-43 pattern 2 and at stages exceeding 2. Thus MAs in axons may be related to the propagation of p-TDP-43 in the nervous tissue in sALS.

The present study is the first to have demonstrated presynaptic aggregates of p-TDP-43 touching or located around neurons of the red nucleus and in the reticular formation of the medulla oblongata in sALS. It has been reported that the axons touching the neurons of the red nucleus originate from the dentate nucleus and motor cortex [21, 22], and that the axons surrounding the neurons in the reticular formation, spinal cord, sensory cranial nuclei, cerebral cortex and cerebellar nuclei [22]. It is unclear why only these axons have p-TDP-43 aggregates in their terminals in SALS, and how these aggregates are formed there.

Although the neurons in the red nucleus are not motor neurons, from the viewpoint of p-TDP-43 propagation, $17 \%$ of the neurons surrounded by presynaptic p-TDP-43 aggregates showed NCIs in their cytoplasm, whereas no aggregates were found at the postsynapse in these neurons. These findings appear to argue against direct transmission of p-TDP-43 across synapses in sALS. However, the neurons surrounded by presynaptic p-TDP-43 aggregates showed loss of p-i-TDP-43 immunoreactivity in the nucleus. Loss of p-i-TDP-43 from the nucleus has been reported to correlate with neuron loss $[9,23-25]$.

Conclusively, two types of axonal p-TDP-43 aggregates - MAs and GRAs - are known to exist predominantly in the facial and hypoglossal nuclei and anterior horn cells of patients with sALS. These aggregates may influence the function of the neurons, and presynaptic aggregation of the protein induces loss of p-i-TDP-43 in the nucleus of postsynaptic neurons. 


\section{Acknowledgements}

The authors are grateful to Ms. Mika Asakawa and Ms. Yayoi Uehara of the Division of Neuropathology, Department of Brain Disease Research, Shinshu University School of Medicine, Nagano, Japan, for their excellent technical assistance. Professor Shu-ichi Ikeda, Dr. Tsuneaki Yoshinaga and Dr. Katsuya Nakamura of the Department of Medicine (Neurology and Rheumatology), Shinshu University School of Medicine, Nagano, Japan, Dr. Kazuma Kaneko, Department of Neurology, Azumino Red Cross Hospital, Azumino, Nagano, Japan, and Dr. Yasuhiro Sakai of the Department of Molecular Pathology, Shinshu University School of Medicine, Nagano, Japan for their providing clinical and pathological information of the patients. The authors are also indebted to Associate Professor Sachiko Takahama of the Faculty of Human Sciences, University of East Asia, Yamaguchi, Japan and Mr. Masayuki Yamada of the Office of Data Science, Kissei pharmaceutical Co., LTD., Tokyo, Japan for their assistance in the statistical analysis.

\section{Funding}

This work was supported in part by grants from the Collaborative Research Project of the Brain Research Institute, Niigata University, Niigata, Japan (2015- \#2702) and the JSPS KAKENHI (Grant-in-Aid for Scientific Research [C] No.15K06754 and [B] No. 25293449).

\section{Author contributions}

Study design: Kiyomitsu Oyanagi

Autopsy findings: Toshitsugu Nakamura, Masahide Watanabe, Kayoko Higuchi, Kouji Asano, Hiroyuki Kanno, Jun Nakayama 
Clinical information: Akiyo Hineno, Takuji Yasude, Hiroyuki Yahikozawa, Katsuhiko Kayanuma, Hideo Makishita, Shinji Ohara, Takao Hashimoto, Toshiaki Sakai

Immunohistochemical and electron microscopic observation: Tomoya Onozato, Asa Nakahara, Emi Suzuki-Kouyama, Tomoyo Hashimoto

\section{Conflict of interest}

The authors have no conflict of interest directly relevant to the content of this article.

\section{References}

1. Arai T, Hasegawa M, Akiyama H, Ikeda K, Nonaka T, Mori H, Mann D, Tsuchiya K, Yoshida M, Hashizume Y, Oda T. TDP-43 is a component of ubiquitin-positive tau-negative inclusions in frontotemporal lobar degeneration and amyotrophic lateral sclerosis. Biochem Biophys Res Commun 2006; 351: 602-11

2. Mackenzie IR, Neumann M, Baborie A, Sampathu DM, Du Plessis D, Jaros E, Perry RH, Trojanowski JQ, Mann DMA, Lee VMY. A harmonized classification system for FTLD-TDP pathology. Acta Neuropathol 2011; 122: 111-3

3. Mackenzie IR, Rademakers R, Neumann M. TDP-43 and FUS in amyotrophic lateral sclerosis and frontotemporal dementia. Lancet Neurol 2010; 9: 995-1007

4. Neumann M, Sampathu DM, Kwong LK, Truax AC, Micsenyi MC, Chou TT, Bruce J, Schuck T, Grossman M, Clark CM, McCluskey LF, Miller BL, Masliah E, Mackenzie IR, Feldman H, Feiden W, Kretzschmar HA, Trojanowski JQ, Lee VMY. Ubiquitinated TDP-43 in frontotemporal lobar degeneration and amyotrophic lateral sclerosis. Science 2006; 314: 130-3

5. Strong MJ, Hortobágyi T, Okamoto K, Kato S. Amyotrophic lateral sclerosis, primary lateral sclerosis and spinal muscular atrophy. In Neurodegeneration Eds. DW Dickson, RO Weller, 2nd edn. 
West Sussex: Wiley-Blackwell, 2011: 418-33

6. Chen-Plotkin AS, Lee VM, Trojanowski JQ. TAR DNA-binding protein 43 in neurodegenerative disease. Nat Rev Neurol 2010; 6: 211-20

7. Janssens J, Broeckhoven CV. Pathological mechanisms underlying TDP-43 driven neurodegeneration in FTLD-ALS spectrum disorders. Hum Mol Genet 2013; 22: 77-87

8. Szafranski K, Abraham KJ, Mekhail K. Non-coding RNA in neural function, disease, and aging. Front Genet 2015; 87: 1-16

9. Brettschneider J, Arai K, Tredici KD, Toledo JB, Robinson JL, Lee EB, Kuwabara S, Shibuya K, Irwin DJ, Fang L, Von Deerlin VMV, Elman L, McCluskey L, Ludolph AC, Lee VMY, Braak H, Trojanowski JQ. TDP-43 pathology and neuronal loss in amyotrophic lateral sclerosis spinal cord. Acta neuropathol 2014; 128: 423-37

10. Hasegawa M, Arai T, Nonaka T, Kametani F, Yoshida M, Hashizume Y, Beach TG, Buratti E, Baralle F, Morita M, Nakano I, Oda T, Tsuchiya K, Akiyama H. Phosphorylated TDP-43 in frontotemporal lobar degeneration and ALS. Ann Neurol 2008; 64: 60-70

11. Ludolph AC, Brettschneider J. TDP-43 in amyotrophic lateral sclerosis - is it a prion disease? Eur J Neurol 2015; 22: 753-61

12. Geser F, Lee VMY, Trojanowski JQ. Amyotrophic lateral sclerosis and fronttemporal lobar degeneration: A spectrum of TDP-43 proteinopathies. Neuropathol 2010; 30: 103-112

13. Nishihira Y, Tan C-F, Onodera O, Toyoshima Y, Yamada M, Morita T, Nishizawa M, Kakita A, Takahashi H. Sporadic amyotrophic lateral sclerosis: two pathological patterns shown by analysis of distribution of TDP-43-immunoreactive neuronal and glial cytoplasmic inclusions. Acta Neuropathol 2008; 116: 169-82

14. Brettschneider J, Tredici KD, Toledo JB, Robinson JL, Irwin DJ, Grossman M, Suh E, Von Deerlin VMV, Wood EM, Baek Y, Kwong L, Lee EB, Elman L, McCluskey L, Fang L, Feldengut S, 
Ludolph AC, Lee VMY, Braak H, Trojanowski JQ. Stages of pTDP-43 Pathology in Amyotrophic Lateral Sclerosis. Ann Neurol 2013; 74: 20-38

15. Jucker M, Walker LC. Self-propagation of pathogenic protein aggregates in neurodegenerative diseases. Nature 2013; 501: 45-51

16. Kanouchi T, Ohkubo T, Yokota T. Can regional spreading of amyotrophic lateral sclerosis motor symptoms be explained by prion-like propagation? J Neurol Neurosurg Psychiatry 2012; 83: 739-45

17. Nonaka T, Masuda-Suzukake M, Arai T, Hasegawa Y, Akatsu H, Obi T, Yoshida M, Murayama S, Mann DMA, Akiyama H, Hasegawa M. Prion-like properties of pathological TDP-43 aggregates from diseased brains. Cell Rep 2013; 4: 124-34

18. Braak H, Ludolph A, Thal DR, Tredici KD. Amyotrophic lateral sclerosis: dash-like accumulation of phosphorylated TDP-43 in somatodendritic and axonal compartments of somatomotor neurons of the lower brainstem and spinal cord. Acta Neuropathol 2010; 120: 67-74

19. Braak H, Brettschneider J, Ludolph AC, Lee VM, Trojanowski JQ, Tredici KD. Amyotrophic lateral sclerosis-a model of corticofugal axonal spread. Nat Rev Neurol 2013; 9: 708-14

20. Thorpe JR, Tang H, Atherton J, Cairns NJ. Fine structural analysis of the neuronal inclusions of frontotemporal lobar degeneration with TDP-43 proteinopathy. J Neural Transm 2008; 115: $1661-71$

21. Katsumaru H, Murakami F, Wu JY, Tsukahara N. Sprouting of GABAergic Synapses in the Red Nucleus After Lesions of the Nucleus Interpositus in the Cat. J Neurosci 1986; 6: 2864-74

22. Parent A. Carpenter's Human Neuroanatomy 9th edn. Baltimore: Williams \& Wilkins, 1996

23. Oyanagi K, Yamazaki M, Hashimoto T, Asakawa M, Wakabayashi K, Takahashi H. Hippocampal sclerosis in the parkinsonism-dementia complex of Guam: quantitative examination of neurons, neurofibrillary tangles, and TDP-43 immunoreactivity in CA1. Neuropathology 2015; 35: 224-35

24. Sumi H, Kato S, Mochimaru Y, Fujimura H, Etoh M, Sakoda S. Nuclear TAR DNA binding protein 
43 expression in spinal cord neurons correlates with the clinical course in amyotrophic lateral sclerosis. J Neuropathol Exp Neurol 2009; 68: 37-47

25. Highley JR, Kirby J, Jansweijer JA, Webb PS, Hewamadduma CA, Heath PR, Higginbottom A, Raman R, Ferraiuolo L, Cooper-Knock J, McDermott CJ, Wharton SB, Shaw PJ, Ince PG. Loss of nuclear TDP-43 in amyotrophic lateral sclerosis (ALS) causes altered expression of splicing machinery and widespread dysregulation of RNA splicing in motor neurons. Neuropathol Appl Neurobiol. 2014; 40: 670-85

\section{Legends}

Table 1. Clinical and neuropathological features of patients with sporadic amyotrophic lateral sclerosis (sALS) and control subjects.

LMN $=$ lower motor neuron; ALS-D = ALS with dementia; NA = not available.

${ }^{a}$ Ref. [12], ${ }^{b}$ Ref. [13], ${ }^{c}$ Ref. [14].

Table 2. Information of the primary antibodies employed.

Table 3. Phosphorylated TDP-43-immunoreactive neuronal cytoplasmic inclusions, axonal and perineuronal aggregates, and skeletal muscle atrophyin the sALS patients and controls.

$\mathrm{AHC}=$ anterior horn cell; bicep $=$ biceps; delt $=$ deltoid; diaph $=$ diaphragm; $\mathrm{GRA}=$ granuloreticular aggregate hypogl $=$ hypoglossal; $;$ iliops $=$ iliopsoas; int cost $=$ intercostal $;$ intramuscul $=$ intramuscular; $\mathrm{MA}=$ massive aggregate $;$ med $\mathrm{obl}=$ medulla oblongata $;$ mod $=$ moderate $;$ ne $=$ not examined $; \mathrm{PN}=$ perineuronal $;$ PNSA = perineuronal synaptic aggregate $;$ precentr $=$ precentral $;$ pyramid $=$ pyramidal $;$ ret form = reticular formation; $\mathrm{RI}=$ round inclusion; sev $=$ severe $; \mathrm{SG}=$ small granule; $\mathrm{SI}=$ skein-like inclusion; $\mathrm{WM}=$ convolutional white matter; $-=$ absent 
Figure 1. Axonal aggregates of p-TDP-43.

Axonal p-TDP-43-ir aggregates in the intramedullary fibers of hypoglossal nerves (A, C-E, G), intramedullary axons of anterior horn cells $(\mathbf{B}, \mathbf{F}, \mathbf{H}-\mathbf{L})$. Two patterns of axonal p-TDP-43-ir aggregates were observed: dash-like granuloreticular aggregates (GRAs) (A-G), and massive aggregates (MAs) (H-L). GRAs were observed as fine granular or reticular features less than $1 \mu \mathrm{m}$ in diameter present within the circumference of the axon (A-G). GRAs were intermingled with p-neurofilament (NF) (C-E, G). p-NF immunoreactivity was lost in many portions at the inside the axons with GRAs (clear stars), but present in the portions without GRAs (solid stars) (F, G). MAs appeared as ovoid or gourd-shaped bodies less than $10 \mu \mathrm{m}$ in longest diameter and less than $5 \mu \mathrm{m}$ in shortest diameter, being surrounded by $\mathrm{p}$-NF (H, J-L). GRAs and MAs were circumscribed by myelin basic protein (MBP)-ir myelin (B, I). MAs were also immunopositive for ubiquitin (M, P, Q) and p62 (N).

A, H: p-TDP-43 immunohistochemistry, B, I; double immunohistochemistry for p-TDP-43 (brown) and MBP (green). F: double immunohistochemistry for p-TDP-43 (brown) and p-NF (green). Imunofluorescence confocal images of p-NF (green: C, E, G, J, L) and p-TDP-43 (red: D, E, G, K, L) and merged images (co-localization appearing as yellow-orange staining: $\mathbf{E}, \mathbf{G}, \mathbf{L}$ ). Ubiquitin (M) and p62 (N) immunohistochemistry. Imunofluorescence confocal microscopy showing anti-p-TDP-43 (green: $\mathbf{O}$ ), anti-ubiquitin (red: P) and the merged image (co-localization appearing as yellow staining: Q). Scale bars in A, B, F, H, I, M, N = $5 \mu \mathrm{m}, \mathbf{C}-\mathbf{E}, \mathbf{G}, \mathbf{J}-\mathbf{L}, \mathbf{O}-\mathbf{Q}=3 \mu \mathrm{m}, \mathbf{F}$; patient No. 1, B, H-L; patient No. 2, A, C-E, G, M-Q; patient No. 6.

Figure 2. Schematic demonstration of the topography of p-TDP-43-ir NCIs and axonal aggregates. p-TDP-43-ir NCIs (black triangles) were observed in the neurons in the hypoglossal and inferior olivary nuclei and reticular formation. p-TDP-43-ir GRAs (red dots) and MAs (light blue dots) were observed in 
the intramedullary and extramedullary fibers of the hypoglossal nerve (A). p-TDP-43-ir NCIs were observed in the anterior horn cells. GRAs were located in intramedullary and extramedullary axons of the anterior horn cells and lateral corticospinal tracts. MAs were observed in the intramedullary axons of the anterior horn cells within the anterior horn (B). MAs were located within several hundred micrometers from the neurons or origin, whereas GRAs were located within a few millimeters.

A; medulla oblongata of patient No. 1, and B; lumbar spinal cord of patient No. 2. Black triangle $=$ neuron with p-TDP-43-ir-NCI, red dot $=$ p-TDP-43-ir-GRA, and light blue dot $=$ p-TDP-ir-MA. Scale bars in $\mathbf{A}=2 \mathrm{~mm}, \mathbf{B}=1 \mathrm{~mm}$.

Figure 3. p-TDP-43-ir aggregates and clinical course.

Appearance of p-TDP-43-ir axonal MA, GRA and NCIs during the clinical course in the facial nucleus or its axons (A), hypoglossal nucleus or its axons (B) and anterior horn cells or their axons $(\mathbf{C})$. Solid circles indicate the presence, and clear square the absence of aggregates. All the patients showing MAs in axons also had GRAs in axons and NCIs, as indicated by the arrows. Horizontal axes indicate duration of illness (months).

Figure 4. Perineuronal presynaptic aggregates (PNSA) of p-TDP-43 in the red nucleus in sALS patient No.1. Intracytoplasmic and PNSA of p-TDP-43 measuring 2 - $5 \mu$ m (arrowheads: a-c) in the red nucleus (A). Neurons with PNSA showed lack of nuclear immunopositivity for p-i-TDP-43 (clear star), instead of presence of nuclear p-i-TDP-43 in neurons without perineuronal aggregates (solid star) (B). Immunofluorescence confocal microscopy showed that PNSA of p-TDP-43 (red: C, E, F), measuring 2 $5 \mu \mathrm{m}$, were surrounded by synaptophysin (SYP) (green: C, D, F) (merged image shows yellow-orange staining: F). (D-F) are magnified images of the box in (C). G; immunoelectron micrograph of (A). 
p-TDP-43-ir a, b and c are seen as a, b and c in $(\mathbf{G})$. (H) is a magnified image of the box in $(\mathbf{G})$. p-TDP-43-ir PNSA were composed of a granular substance $(\mathbf{H})$. Schematic illustration of neurons with p-TDP-ir-NCI (black triangles) and neurons with p-TDP-ir PNSA (red dots) in the mid brain of patient No. 1 (I). Medial predominance of neurons with p-TDP-ir PNSA is evident in the red nucleus (I). A: p-TDP-43 immunohistochemistry, B: p-i-TDP-43 immunohistochemistry. Imunofluorescence confocal images of SYP (green: C, D, F) and p-TDP-43 (red: C, E, F) and merged images (co-localization appearing as yellow-orange staining: F). G, H: immunoelectron micrograph with p-TDP-43-ir and stained with uranyl-lead. Scale bars in $\mathbf{A}-\mathbf{C}, \mathbf{G}=10 \mu \mathrm{m}, \mathbf{D}-\mathbf{F}, \mathbf{H}=2 \mu \mathrm{m}, \mathbf{I}=5 \mathrm{~mm} . \mathrm{n}=$ nucleus. 


\begin{tabular}{|c|c|c|c|c|c|c|c|c|c|c|c|}
\hline $\begin{array}{l}\text { Patients } \\
\text { and } \\
\text { subjects }\end{array}$ & $\begin{array}{l}\text { Age at death } \\
\text { (years old) }\end{array}$ & Gender & $\begin{array}{l}\text { Duration } \\
\text { of illness } \\
\text { (months) }\end{array}$ & $\begin{array}{l}\text { Site of initial } \\
\text { symptom }\end{array}$ & Fasciculation & $\begin{array}{l}\text { Artificial } \\
\text { ventilation } \\
\text { (months) }\end{array}$ & Cause of death & $\begin{array}{l}\text { Bunina } \\
\text { body }\end{array}$ & $\begin{array}{l}\text { TDP-43 } \\
\text { spectrum }^{\mathrm{a}}\end{array}$ & $\begin{array}{l}\text { TDP-43 neuronal } \\
\text { distribution pattern }\end{array}$ & $\begin{array}{l}\text { Stages of TDP-43 } \\
\text { pathology }\end{array}$ \\
\hline ALS & & & & & & & & & & & \\
\hline 1 & 68 & Male & 6 & Upper & NA & - & Pneumonia & LMN & ALS-D & 2 & 4 \\
\hline 2 & 72 & Female & 6 & Lower & NA & - & Pneumonia & LMN & ALS-D & 2 & 4 \\
\hline 3 & 59 & Male & 8 & Lower & + & - & Pneumonia & LMN & ALS-D & 2 & 2 \\
\hline 4 & 63 & Male & 8 & Lower & + & - & Respiratory failure & LMN & ALS-D & 2 & $2 / 3$ \\
\hline 5 & 74 & Female & 8 & Lower & NA & - & Respiratory failure & LMN & ALS-D & 2 & 3 \\
\hline 6 & 88 & Male & 11 & Lower & + & 2 & Respiratory failure & LMN & ALS-D & 2 & 2 \\
\hline 7 & 60 & Male & 16 & Lower & + & - & Pneumonia & LMN & ALS & 1 & 1 \\
\hline 8 & 69 & Male & 18 & Lower & + & - & Pneumonia & LMN & ALS & 1 & 2 \\
\hline 9 & 60 & Male & 19 & Upper & + & - & Pneumonia & LMN & ALS-D & 2 & 2 \\
\hline 10 & 70 & Male & 19 & Unclear & + & - & Pneumonia & LMN & ALS & 1 & 2 \\
\hline 11 & 86 & Male & 21 & Lower & NA & - & Pneumonia & LMN & ALS & 1 & 1 \\
\hline 12 & 61 & Male & 27 & Upper & NA & - & Respiratory failure & LMN & ALS-D & 2 & 4 \\
\hline 13 & 76 & Female & 31 & Unclear & NA & - & Respiratory failure & LMN & ALS & 1 & $1 / 2$ \\
\hline 14 & 82 & Female & 33 & Upper & + & 1 & Respiratory failure & LMN & ALS & 1 & $2 / 3$ \\
\hline 15 & 65 & Female & 36 & Bulbar & NA & - & Senile pulmonary emphysema & LMN & ALS-D & 2 & $2 / 3$ \\
\hline 16 & 68 & Male & 36 & Lower & NA & - & Pneumonia & LMN & ALS-D & 2 & 3 \\
\hline 17 & 59 & Female & 40 & Upper & NA & - & Pneumonia & LMN & ALS & 1 & 1 \\
\hline 18 & 80 & Female & 44 & Upper & NA & - & Pneumonia & - & ALS & 1 & $1 / 2$ \\
\hline 19 & 73 & Female & 72 & Unclear & NA & - & Respiratory failure & - & ALS & 1 & 1 \\
\hline \multicolumn{12}{|c|}{ Controls } \\
\hline 20 & 65 & Male & - & NA & NA & - & \multicolumn{5}{|c|}{ Acute leukemia, disseminated intravascular coagulation } \\
\hline 21 & 69 & Male & - & NA & NA & - & \multicolumn{5}{|c|}{ Cerebral amyloid angiopathy, cerebral infarct, familial amyloid polyneuropathy } \\
\hline 22 & 74 & Male & - & $\mathrm{NA}$ & NA & - & \multicolumn{5}{|c|}{ Aplastic anemia, diabetes, intracerebral hemorrhage } \\
\hline
\end{tabular}




\begin{tabular}{llllll}
\hline Detection & Antibody type & Clone & Dilution & Increase of antigenicity & Source \\
\hline Phosphorylated TDP-43 (p-TDP-43) & Mouse monoclonal & $11-9$ & $1: 10000$ & Autoclaving & COSMO BIO, Tokyo, Japan \\
Phosphorylated TDP-43 (p-TDP-43) & Rabbit polyclonal & & $1: 3000$ & Boiling & COSMO BIO, Tokyo, Japan \\
Phosphorylation independent TDP-43 (p-i-TDP-43) & Rabbit polyclonal & & $1: 5000$ & Autoclaving & ProteinTech Group, Chicago, USA \\
Ubiquitin & Rabbit polyclonal & & $1: 1000$ & Autoclaving & DakoCytomation, Glostrup, Denmark \\
p62 & Rabbit polyclonal & & $1: 2000$ & Autoclaving & Enzo LifeSciences, Lausen, Switzerland \\
Myeline basic protein (MBP) & Rabibt polyclonal & $1: 400$ & Autoclaving & Abcam. Tokyo, Japan \\
Synaptophysin (SYP) & Mouse monoclonal & $171 \mathrm{~B} 5$ & $1: 200$ & Autoclaving & Medical \& Biological Laboratories, Nagoya, Japan \\
Phosphorylated alpha synuclein & Mouse monoclonal & pSYN\#64 & $1: 20000$ & Formic acid & Wako Pure Chemicals Industries, Osaka, Japan \\
Phosphorylated neurofilament (p-NF) & Mouse monoclonal & SMI31 & $1: 2000$ & Boiling & COVANCE, Berkeley, USA \\
Phosphorylated tau & Mouse monoclonal & AT8 & $1: 3000$ & & Innogenetics, Ghent, Belgium \\
\hline
\end{tabular}




\begin{tabular}{|c|c|c|c|c|c|c|c|c|c|c|c|c|}
\hline \multirow[b]{2}{*}{$\begin{array}{l}\text { Patients } \\
\text { and } \\
\text { subjects }\end{array}$} & \multicolumn{4}{|c|}{ Neuronal cytoplasmic inclusions } & \multirow[b]{2}{*}{$\begin{array}{l}\text { Red } \\
\text { nucleus }\end{array}$} & \multicolumn{5}{|c|}{ Axonal aggregates } & \multicolumn{2}{|c|}{ PN aggregates } \\
\hline & $\begin{array}{l}\text { Motor } \\
\text { cortex }\end{array}$ & $\begin{array}{l}\text { Facial } \\
\text { nucleus }\end{array}$ & $\begin{array}{l}\text { Hypogl } \\
\text { nucleus }\end{array}$ & $\begin{array}{l}\text { Anterior } \\
\text { horn }\end{array}$ & & $\begin{array}{l}\text { Precentr } \\
\text { gyrus } \\
\text { WM }\end{array}$ & $\begin{array}{l}\text { Pyramid } \\
\text { tract }\end{array}$ & $\begin{array}{l}\text { Fibers of } \\
\text { facial } \\
\text { nerve }\end{array}$ & $\begin{array}{l}\text { Fibers of } \\
\text { hypogl } \\
\text { nerve }\end{array}$ & $\begin{array}{l}\text { Axon of } \\
\text { AHC }\end{array}$ & $\begin{array}{l}\text { Red } \\
\text { nucleus }\end{array}$ & $\begin{array}{l}\text { Ret form } \\
\text { of } \\
\text { med obl }\end{array}$ \\
\hline \multicolumn{13}{|l|}{$\overline{\text { ALS }}$} \\
\hline 1 & RI & RI & SG & RI, SG & RI & GRA & - & GRA & GRA,MA & GRA,MA & PNSA & PNSA \\
\hline 2 & - & ne & ne & SI, SG & ne & - & GRA & ne & ne & GRA,MA & ne & ne \\
\hline 3 & - & ne & SI & SI, RI & RI & - & - & ne & - & GRA & - & - \\
\hline 4 & - & - & SI, RI & SI, RI & - & - & - & - & - & - & - & - \\
\hline 5 & - & - & SI & SI & RI & - & - & - & - & - & PNSA & - \\
\hline 6 & SG & RI, SG & RI, SG & SI, RI & RI & GRA & - & GRA,MA & GRA,MA & GRA,MA & PNSA & - \\
\hline 7 & - & ne & SI & SI & - & - & - & ne & GRA & - & - & - \\
\hline 8 & - & ne & SG & SG & - & - & - & ne & GRA & GRA & - & - \\
\hline 9 & - & SI, SG & SI, SG & SI, SG & - & - & - & GRA & GRA & GRA & - & - \\
\hline 10 & SG & ne & SG & SG & RI & - & - & ne & - & - & - & - \\
\hline 11 & - & ne & SI & SI & ne & - & - & ne & - & GRA & ne & - \\
\hline 12 & - & RI, SG & RI, SG & SI, RI, SG & - & - & - & GRA & GRA & GRA & - & - \\
\hline 13 & ne & ne & ne & SI & ne & ne & - & ne & ne & GRA & ne & ne \\
\hline 14 & ne & ne & ne & RI & ne & ne & - & ne & ne & - & ne & ne \\
\hline 15 & ne & ne & SG & SI & ne & ne & - & ne & - & - & ne & - \\
\hline 16 & SG & SG & SG & SI, RI & - & - & - & - & - & - & - & - \\
\hline 17 & ne & ne & SI & SI & ne & ne & - & ne & - & - & ne & - \\
\hline 18 & ne & ne & ne & SG & ne & ne & - & ne & ne & - & ne & ne \\
\hline 19 & ne & SI & - & SI, SG & - & ne & - & - & - & - & - & - \\
\hline \multicolumn{13}{|c|}{ Controls } \\
\hline 20 & - & - & - & - & - & - & - & - & - & - & - & - \\
\hline 21 & - & - & - & - & - & - & - & - & - & - & - & - \\
\hline 22 & - & - & - & - & - & - & - & - & - & - & - & - \\
\hline
\end{tabular}




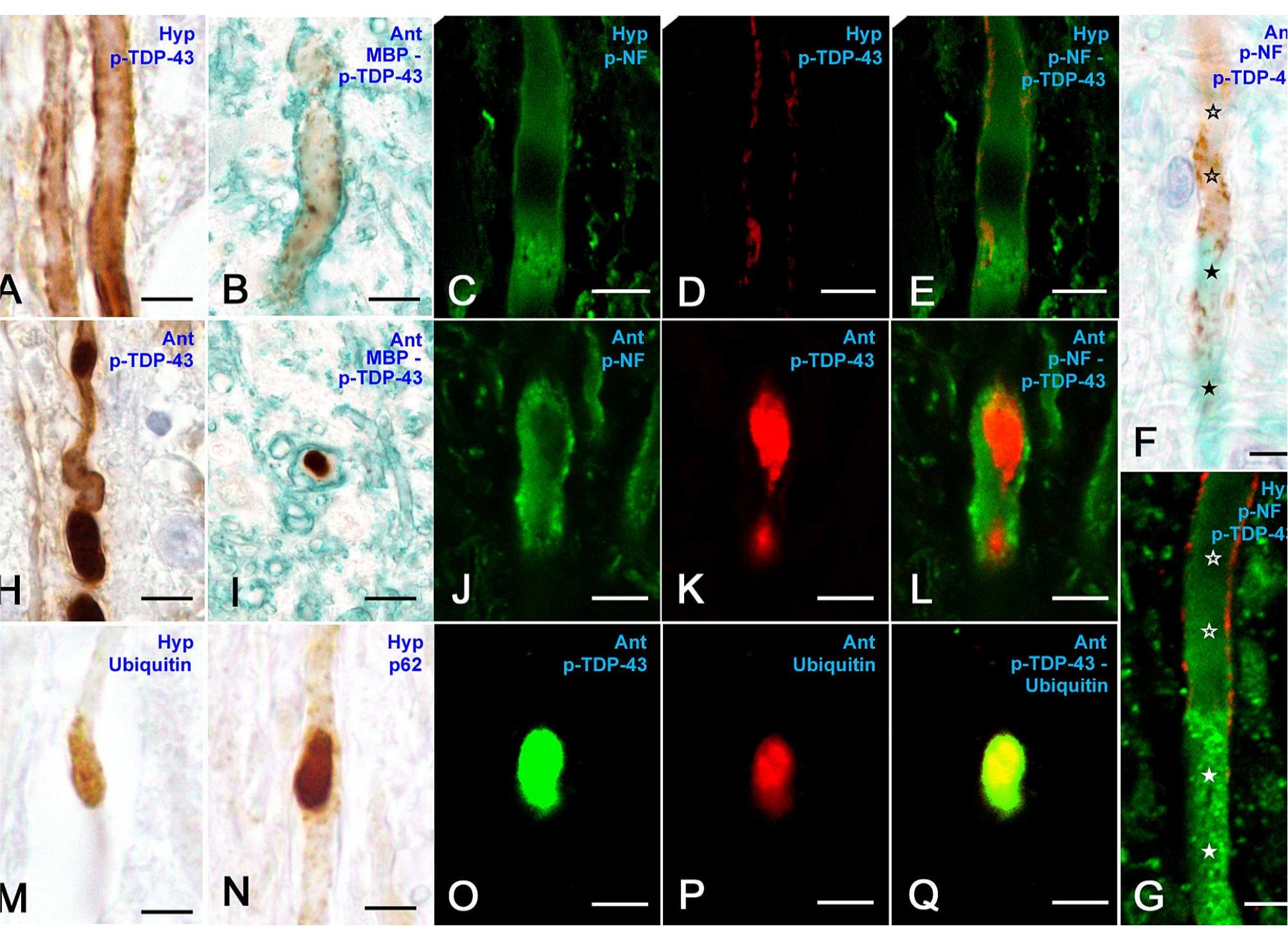



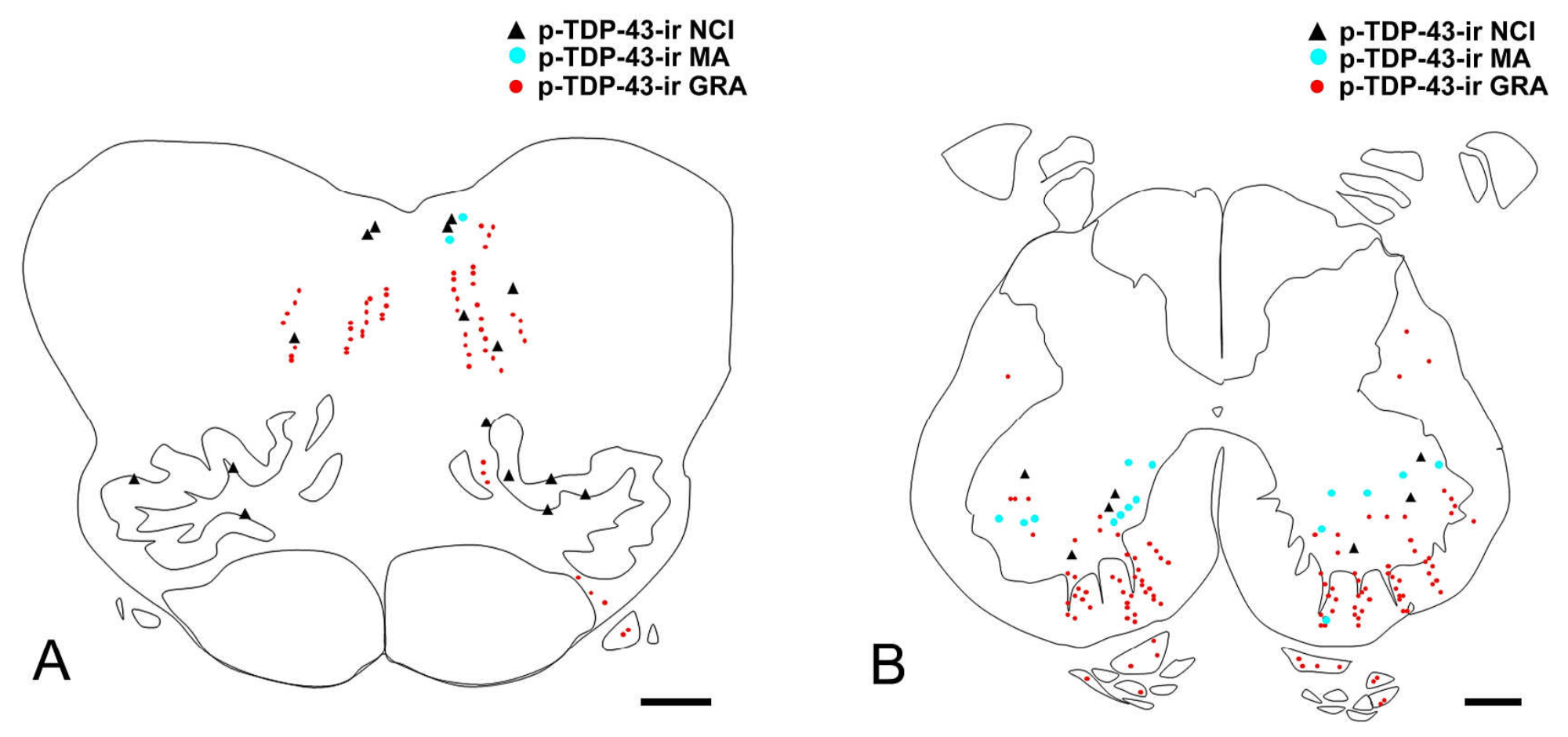

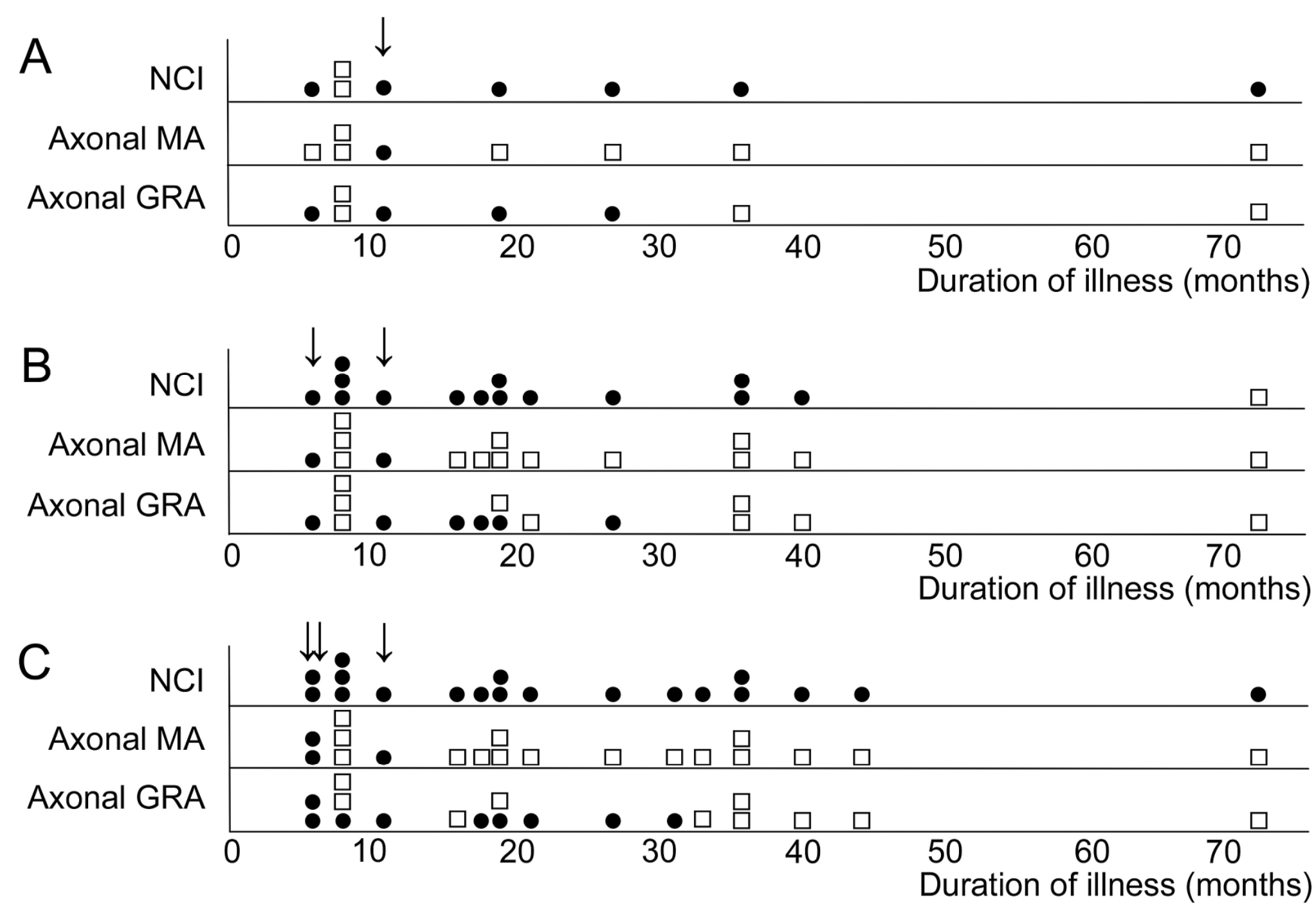


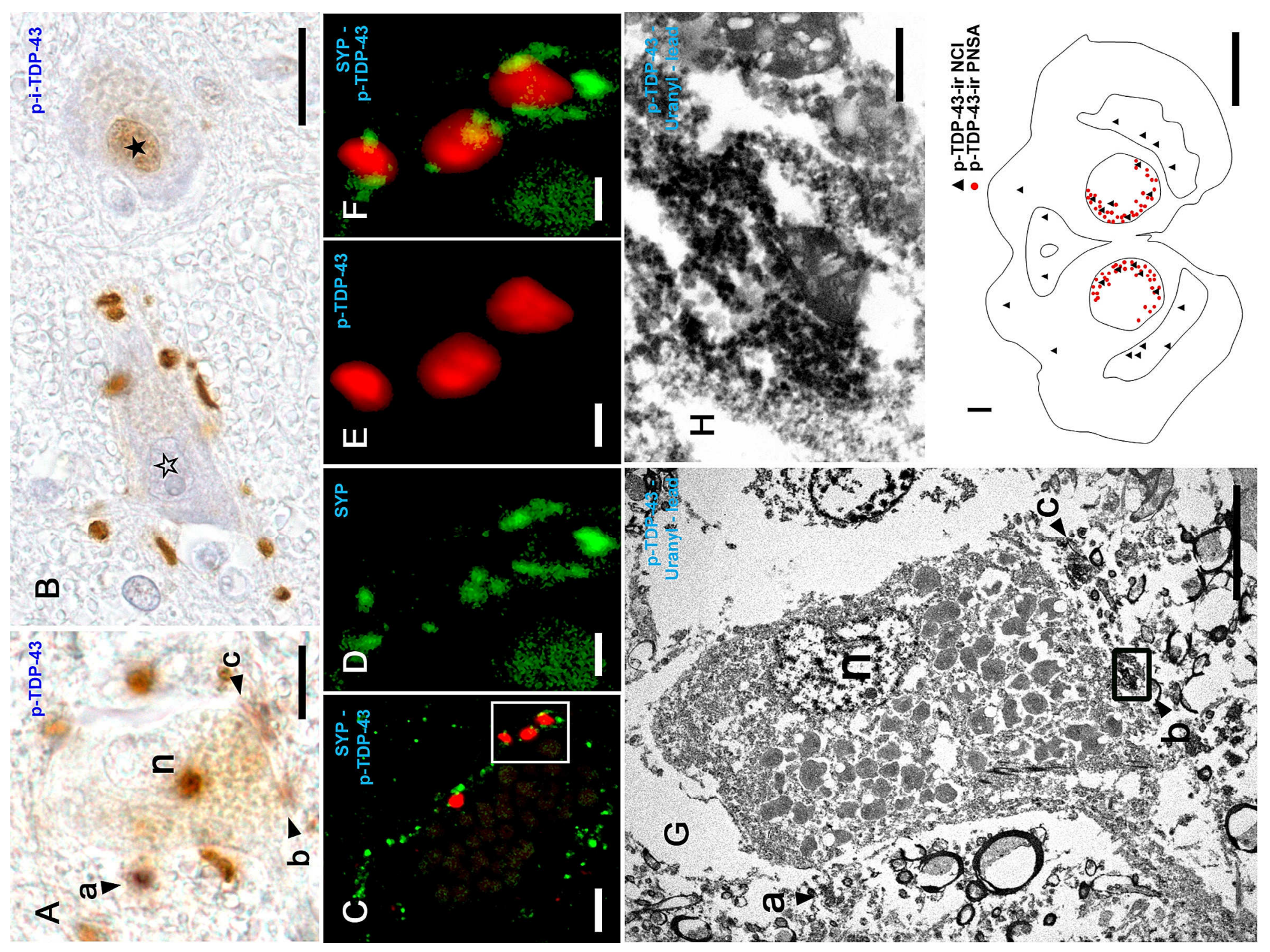

